\title{
UNDERSTANDING CHANGES IN PERIOD RATIOS
}

\author{
J. O. PETERSEN \\ Copenhagen University Observatory, Copenhagen, Denmark
}

The discrepancies between observed and theoretically calculated period ratios of the double mode, classical Cepheids and other groups of Cepheid type variables have played a prominent role in investigations of the important physical characteristics of these stars during the last twenty years. Today, there is growing consensus that the drastic increases in astrophysical opacities proposed by Simon (1982) provide the correct solution of all the period ratio problems (e.g. Petersen, 1989; Iglesias and Rogers, 1992; Moskalik et al., 1991).

It seems at first strange that the optimal opacity change (see Andreasen, 1988) can produce both the decrease in period ratio for double mode classical Cepheids and the increased period ratio in models of $\delta$ Scuti stars. Here we use $\kappa$-effect-functions (Refsdal and Stabell, 1972) to provide both a detailed description of the effects of opacity changes and an improved and more direct understanding of these effects.

Denoting the period ratio $\Pi_{10}=\Pi_{1} / \Pi_{0}$, the $\kappa$-effect-function for the period ratio is defined by

$$
\varphi(\log T)=\frac{\Delta \Pi_{10}}{\Delta \log \kappa \Delta \log T}
$$

Thus $\varphi$ is the increase in period ratio per unit increase in $\log \kappa$ and unit temperature interval $\Delta \log T$ (see Refsdal and Stabell for details). Fig. 1 compares the $\kappa$-effect-functions for models of a typical double mode Cepheid of period 2.82 days and a $\delta$ Scuti star of period 0.127 days. It is seen that $\varphi$ behaves very differently in the two cases in the temperature interval $\log T=$ $5.2-5.8$, which is relevant for realistic (and the optimal) opacity changes. In the case of the double mode Cepheid $\varphi$ is negative in this temperature interval. This means that an opacity increase results in a decrease in the period ratio. For the $\delta$ Scuti model $\varphi$ is seen to be positive except in a small region. Therefore, the same opacity increase now results in an increased period ratio.

Very recently, Iglesias and Rogers (1992, Fig. 24) gave detailed corrections to the Cox-Tabor opacities. Using these correction factors and the $\kappa$-effectfunctions shown in Fig. 1, we can calculate the resulting period ratio changes. We consider the model as consisting of (many) layers of thickness $\Delta \log T$. Then Eq. (1) tells us that each layer produces a correction to the period ratio of the model of $\Delta \Pi_{10}=\varphi \Delta \log \kappa \Delta \log T$, where $\varphi$ is given in Fig. 1 and $\Delta \log \kappa$ can be determined from the information given in Iglesias and 


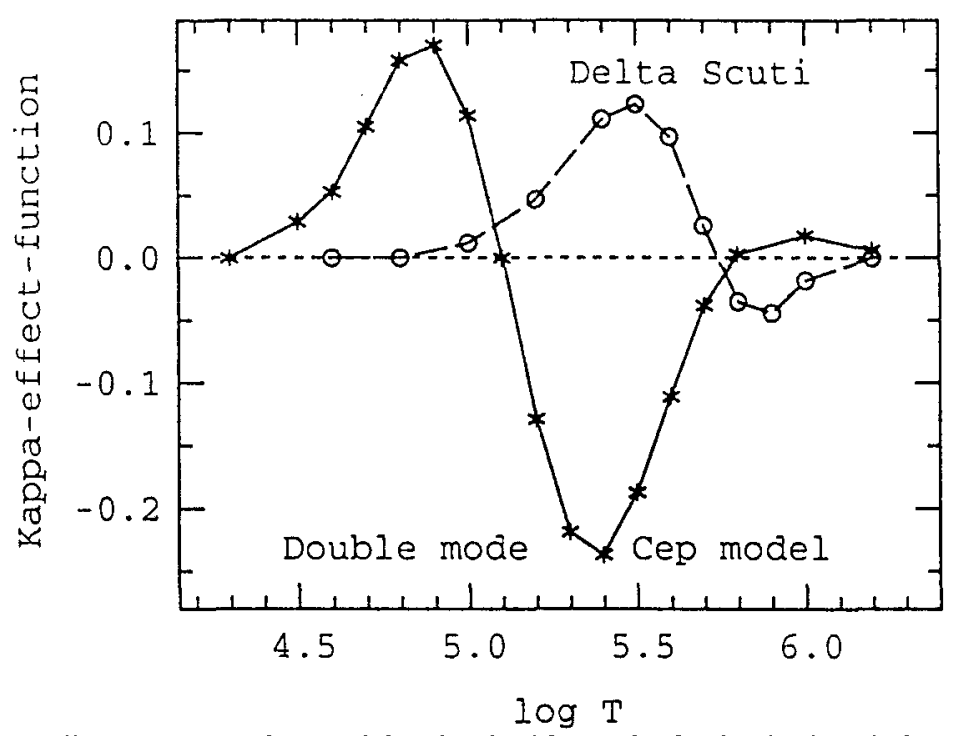

Fig. 1. $\kappa$-effect-functions for models of a double mode Cepheid of period 2.82 days and a $\delta$ Scuti star of period of 0.127 days.

Rogers. For the Cepheid case we obtain a total correction to the period ratio of $\Delta \Pi_{10}=-0.037$. This compares favourably with the difference between the observed values close to 0.70 and the theoretical values based upon the Los Alamos standard opacities of about 0.74 . In the $\delta$ Scuti case we find $\Delta \Pi_{10}=+0.016$. This is in good agreement with the difference between observed period ratios close to 0.773 and the values of about 0.76 based upon the Cox-Tabor opacities.

Using the $\kappa$ - $\epsilon f f \epsilon c t$-functions given in Fig. 1 , it is very easy to calculate the effects resulting from arbitrary opacity changes. And besides, these curves give a natural feeling for the complicated relations between opacities and period ratios. Consider e.g. an opacity increase in the temperature interval $\log T=4.5-5.1$. Fig. 1 immediately tells us that in the Cepheid case this increase will result in an increase in the resulting period ratio. In the $\delta$ Scuti case an opacity increase in $\log T=4.5-5.1$ is seen to have a very small effect. We conclude that $\kappa$-effect-functions for period ratios give an improved understanding of the relations between opacities and period ratios, and that they are very useful for discussions of improved opacities.

This work has been supported by the Danish Natural Science Research Council through grant 11-9024.

\section{References}

Andreasen, G. K.: 1988, Astronomy and Astrophysics 201, 72.

Iglesias, C. A. and Rogers, F. J.: 1992, preprint. 
Moskalik, P., Buchler. J. R. and Marom, A.: 1991, preprint.

Petersen, J. O.: 1989. Astronomy and Astrophysics 226, 151.

Refsdal, S. and Stabell, R.: 1972, Astronomy and Astrophysics 20, 19.

Simon, N. R.: 1982, Astrophysical Journal, Letters to the Editor 260. L87. 